Award, the Canadian Anaesthetists' Society most definitely does include the promotion and support of scientific research amongst its priorities.

In practical terms, the Research Award will provide important "start up" funds for a new investigator. Ideally, the Award will be made to applicants who have acquired, through special training and research experience, skills which will allow them to begin careers as researchers in anaesthesia; Canadian researchers in Canadian research programs.

We join with all members of the Canadian Anaesthetists' Society in extending our very best wishes to the first recipient of the Canadian Anaesthetists' Society Research Award as he embarks on what we hope will be a long and successful career in anaesthesia research.

\section{Le prix pour la recherche décerné par la Société canadienne des anesthésistes}

Félicitations au premier récipiendaire du prix pour la recherche décerné par la Société canadienne des Anesthésistes, le docteur Howard J. Nathan, du département d'anesthésie de l'Université d'Ottawa. Le projet du docteur Nathan était intitulé "Agents anesthésiques et ischémie myocardique." Le prix de $\$ 20,000$ a été présenté à la réunion annuelle tenue à Toronto au mois de juin.

Une reconnaissance spéciale est aussi due aux administrateurs du Fonds de recherche de la Société canadienne des anesthésistes qui, en 1979, ont eu la prévoyance d'établir ce Fonds avec l'approbation et l'encouragement du Conseil de la Société canadienne des anesthésistes. Le docteur Gordon Sellery, au nom des administrateurs de ce Fonds, en a été un promoteur infatigable. On lui doit un vote spécial de remerciement pour sa persévérance. L'octroi de ce premier prix pour la recherche est symbolique des efforts spéciaux que le docteur Gordon Sellery a accomplis au nom de tous les anesthésistes canadiens.

Depuis l'annonce que le premier prix pour la recherche sera attribué en 1985 et la publication de la demande de projets, l'intérêt dans le Fonds de recherche a augmenté. Pendant la dernière année, les dons au Fonds de recherche ont augmenté d'une façon considérable. L'argent confié en fidéicommis par le Fonds a doublé dans les douze derniers mois. En plus de la contribution faite par des centaines de membres de la société, plusieurs divisions ont aussi ajouté des contributions spéciales. Parmi ces divisions, on inclut celle de l'Ontario (deux fois), Terre-Neuve, Saskatchewan, Alberta et Manitoba. De plus, on a assisté à une augmentation d'intérêt de certaines corporations à contribuer par des dons à ce Fonds de recherche. Au cours de l'année précédente, le nombre de ces corporations a augmenté de 3 à 17 .

A la réunion annuelle de 1985 , la Société internationale de recherche en Anesthésie (the International Anesthesia Research Society) a présenté la somme de $\$ 5,000$ (U.S.) au Fonds de recherche et ce en reconnaissance de la participation antérieure et actuelle des membres de la Société canadienne des anesthésistes dans la Société internationale de recherche en anesthésie (IARS).

L'argent tenu dans le Fonds a doublé au cours de la dernière année. Il doit doubler encore avant qu'un capital suffisant $(\$ 200,000)$ soit disponible afin de générer un prix annuel de $\$ 20,000$ uniquement à partir du revenu investi.

On est confiant que l'intẻrêt récent soulevé et le support financier pour le Fonds de recherche seront soutenus tant dans les cercles privés qu'au sein des corporations. Le Canadiens doivent être fiers et à juste titre de la recherche médicale conduite dans leur pays. La recherche en anesthésie au Canada est florissante. Un nombre croissant de projets de recherche issus de centres canadiens sont soumis pour des présentations à la réunion annuelle de la Société canadienne des anesthésistes. Aussi, plusieurs articles sont soumis par des départements canadiens en vue de publication dans le Journal de la Société Canadienne des Anesthésistes et dans d'autres journaux à travers le monde

Malgré ces signes optimistes on doit se rendre compte que le nombre des investigateurs de carrière et de chercheurs en formation en anesthésie au 
Canada est en fait très petit. Le comité de recherche de l'Association canadienne des départements universitaires d'anesthésie (A.C.U.D.A.) en 1984, a identifié uniquement cinq postes d'associés de recherche remplis par des anesthésistes à travers le Canada. Les restrictions monétaires pour les projets de recherche constituent la restriction majeure tant en anesthésie que dans d'autres spécialités.

Le prix pour la recherche de la Société canadienne des anesthésistes contribuera tant au point de vue symbolique que pratique à résoudre ces problèmes. En établissant ce Fonds en 1979 et en accordant le premier prix en 1985, la Société canadienne des anesthésistes s'est déclarée en faveur de la recherche en anesthésie. Par son congrès annuel, son journal scientifique qui est actuellement à sa 32e année de publication et son prix pour la recherche, la Société canadienne des Anesthésistes met certainement en priorité la promotion et le support de la recherche scientifique.

En terms pratiques, le prix pour la recherche sera un point de départ important pour des nouveaux chercheurs. Idéalement, ce prix sera offert aux candidats qui ont acquis, par un entraînement spécial et une expérience en recherche, des aptitudes qui leur permettent de débuter une carrière en recherche en anesthésie. Il faut former des chercheurs canadiens dans des programmes canadiens.

On se joint à tous les membres de la Société canadienne des anesthésistes pour transmettre nos meilleurs souhaits au premier récipiendaire du prix pour la recherche de la Société canadienne des anesthésistes alors qu'il débute dans ce qu'on souhaite être une longue carrière pleine de succès en recherche en anesthésie. 